\title{
Ectopic Fat is Linked to Prior Cardiovascular Events in Men With HIV
}

\author{
Orlando Gabriella, PhD, MD, * Guaraldi Giovanni, MD, * Zona Stefano, MD, * Carli Federica, MD, * \\ Bagni Pietro, MD, * Menozzi Marianna, MD, * Cocchi Stefania, PhD, MD, * Scaglioni Riccardo, MD, $\dagger$ \\ Ligabue Guido, MD, † and Raggi Paolo, PhD, MD $\neq$
}

\begin{abstract}
Epicardial Adipose Tissue (EAT) has been associated with adverse cardiovascular events in the general population. We studied the association of general adiposity measures (body mass index, waist circumference) and ectopic adipose tissue [visceral adipose tissue (VAT); liver fat (LF); EAT) with prevalent cardiovascular disease (CVD) (prior myocardial infarction, coronary revascularization, stroke, peripheral vascular disease] in 583 HIV-infected men. VAT, EAT, and LF (liver/spleen attenuation ratio $<1.1$ ) were measured by computed tomography. Patients' mean age was $48.5 \pm$ 8.1 years, prior CVD was present in $33(5.7 \%)$ patients. Factors independently associated with CVD on multivariable analyses were age [incidence-rate ratio $(\mathrm{IRR})=1.07,95 \%$ confidence interval $(\mathrm{CI}): 1.02$ to 1.12], smoking ( IRR $=2.70,95 \% \mathrm{CI}: 1.22$ to 6.01 ), Center for Disease Control group C (IRR $=3.09,95 \%$ CI: 1.41 to 6.76 ), EAT $\left(\mathrm{IRR}=1.13,95 \% \mathrm{CI}\right.$ : 1.04 to 1.24 , per $\left.10 \mathrm{~cm}^{3}\right), \mathrm{LF}(\mathrm{IRR}=1.17,95 \%$ CI: 1.04 to 1.32 ), and VAT (IRR $=1.05,95 \%$ CI: 1.00 to 1.10 , per $10 \mathrm{~cm}^{3}$ ). Ectopic fat but not general adiposity measures were associated with prevalent CVD in men with HIV.
\end{abstract}

Key Words: HIV, cardiovascular disease, ectopic fat measurements (J Acquir Immune Defic Syndr 2012;59:494-497)

\section{INTRODUCTION}

In the general population visceral adipose tissue (VAT) has been demonstrated to correlate with traditional cardiovascular risk factors: total cholesterol, low high-density lipoprotein cholesterol, triglycerides, Apolipoprotein B, blood pressure, insulin resistance, and C-reactive protein. ${ }^{1-3}$ It is believed that VAT releases free fatty acids into the hepatic circulation, thus stimulating the release of Apolipoprotein B-containing lipoproteins, reducing insulin sensitivity, and increasing plasma glucose values. ${ }^{4,5}$ Furthermore, VAT releases several cytokines that may be involved in the development of atherosclerosis. ${ }^{6}$ Epicardial adipose tissue (EAT) shares a common embryological origin with VAT and is metabolically very active, secreting

Received for publication November 2, 2011; accepted January 17, 2012. From the *Department of Medicine and Medical Specialties, University of Modena and Reggio Emilia, Modena, Italy; †Department of Imaging, University of Modena and Reggio Emilia, Modena, Italy; and $\vdots$ Department of Medicine, Division of Cardiology, Emory University, Atlanta, GA.

The authors have no funding or conflicts of interest to disclose.

Correspondence to: Guaraldi Giovanni, MD, Dipartimento di Mdedicine e

Specialità Mediche, Università di Modena e Reggio Emilia, Via del Pozzo

71, 41100 Modena, Italy (e-mail: giovanni.guaraldi@unimore.it).

Copyright (C) 2012 by Lippincott Williams \& Wilkins proinflammatory cytokines at a higher rate than subcutaneous and omental fat. ${ }^{7-9}$ It is postulated that EAT may influence coronary atherosclerosis development via paracrine and systemic effects; EAT has been associated with incident coronary events in the general population ${ }^{10}$ and with VAT, fasting glucose and insulin, ${ }^{11}$ and subclinical atherosclerosis ${ }^{12}$ in HIV. We evaluated the association of adiposity measures [body mass index $(\mathrm{BMI})$ ] and regional and ectopic fat [waist circumference, VAT, EAT, and liver fat (LF)] with prevalent cardiovascular disease (CVD) in 583 men with HIV infection.

\section{METHODS}

\section{Subjects}

Cross-sectional observational study of 583 consecutive HIV-infected men receiving stable antiretroviral therapy (ART) for at least 6 months referred to the Metabolic Clinic of the University of Modena and Reggio Emilia, Italy, between January 1, 2005, and December 31, 2009.

\section{Assessments}

Demographic characteristics, HIV infection history [Center for Disease Control (CDC) classification, type and duration of ART (nucleoside reverse transcriptase inhibitor, nonnucleoside reverse transcriptase inhibitor, protease inhibitor)], smoking, physical activity, blood pressure, weight, and height were collected during patient encounters. Waist circumference was averaged over 3 measurements and body mass index was calculated as weight $(\mathrm{kg}) /$ height $(\mathrm{m})^{2}$. Laboratory analyses included the following: CD4+ count, plasma HIV-1 RNA, fasting total cholesterol, LDL cholesterol, triglycerides, high-density lipoprotein cholesterol, apolipoprotein A1 (Apo A), apolipoprotein B (Apo B), blood glucose, and insulin. The homeostasis model assessment of insulin resistance (HOMA-IR) index was calculated using the algorithm HOMA-IR $=[$ glucose, $\mathrm{mmo} / \mathrm{L} \times$ $0.05551) \times($ insulin, $\mathrm{mU} / \mathrm{L})] / 22.5 \mathrm{~g}$. Lipodystrophy was diagnosed according to established criteria. ${ }^{13}$ A 10 -year risk of CVD was calculated with the modified Framingham equation. ${ }^{14}$

Prior cardiovascular events were defined as myocardial infarction (MI), coronary revascularization, stroke, peripheral vascular disease that occurred within a 5-year period of the computed tomography (CT) assessment of ectopic fat. Cardiovascular events reported by the patients or obtained from the Metabolic Clinic charts were verified using the same classification criteria used for the D:A:D cohort; indeed, a subset of the Metabolic Clinic patients is enrolled in the D.A.D. In particular, 
TABLE 1. Clinical and Demographic Characteristics of the Sample

\begin{tabular}{|c|c|c|c|}
\hline Variables & $\begin{array}{c}\text { CVD Yes (33, 5.7\%) Median (IQR) } \\
\text { or Mean (SD) }\end{array}$ & $\begin{array}{c}\text { CVD No (550, } 94.3 \%) \text { Median (IQR) } \\
\text { or Mean (SD) }\end{array}$ & $P$ \\
\hline HIV duration, mo & $194.5(157.5-249)$ & $181(130-235.5)$ & 0.091 \\
\hline CD4 nadir, cells $/ \mu \mathrm{L}$ & $190(50-241)$ & $160(60-274)$ & 0.836 \\
\hline Past IVD use, n (\%) & $9(27.3)$ & $142(25.8)$ & 0.853 \\
\hline No physical activity, n (\%) & $22(66.7)$ & $293(54.3)$ & 0.154 \\
\hline Alcohol consumption, n (\%) & $16(48.5)$ & $221(40.7)$ & 0.378 \\
\hline Waist circumference, $\mathrm{cm}$ & $93.0(7.7)$ & $88.7(9.8)$ & 0.004 \\
\hline BMI & $25.7(3.0)$ & $24.2(3.6)$ & 0.024 \\
\hline $\mathrm{VAT}, \mathrm{cm}^{3}$ & $207.2(85.7)$ & $145.0(72.7)$ & $<0.0001$ \\
\hline EAT, $\mathrm{cm}^{3}$ & $106(81-169)$ & $81(56-103)$ & $<0.0001$ \\
\hline NNRTIs exposure, months & $14(0-45)$ & $22(0-54)$ & 0.326 \\
\hline PIs exposure, months & $59(33-94)$ & $39(11-77)$ & 0.077 \\
\hline Statin use $\mathrm{n}(\%)$ & $22(66.7)$ & $53(9.7)$ & $<0.001$ \\
\hline No lipodystrophy, n (\%) & 0 & $77(17)$ & 0.024 \\
\hline Lipoatrophy, n (\%) & $5(20)$ & $187(41.3)$ & 0.037 \\
\hline Central fat accumulation, $\mathrm{n}(\%)$ & $5(20)$ & $43(9.5)$ & 0.094 \\
\hline Mixed form, n (\%) & $15(60)$ & $146(32.2)$ & 0.004 \\
\hline Diastolic pressure, $\mathrm{mmHg}$ & $78.2(11.8)$ & $78.6(11.2)$ & 0.724 \\
\hline Systolic pressure, $\mathrm{mmHg}$ & $123.0(15.4)$ & $121.5(15.6)$ & 0.547 \\
\hline Framingham risk score, $\%$ & $12(10-16)$ & $8(4-12)$ & $<0.0001$ \\
\hline Metabolic syndrome, n (\%) & $7(21.9)$ & $113(21.7)$ & 1 \\
\hline
\end{tabular}

ApoB/ApoA, apolipoprotein A/apolipoprotein B ratio; HCV, hepatitis C virus; HDL, high-density lipoprotein; HOMA-IR, homeostatis model of assessment of insulin resistance; IQR, interquartile range; IVD, intravenous drug; NRTI, nucleoside reverse transcriptase inhibitor; NNRTI, nucleoside reverse transcriptase inhibitor; PI, protease inhibitor.

MI's are classified according to criteria applied in the World Health Organization Monitoring Trends and Determinants in Cardio-vascular Disease study. ${ }^{15}$

CT scans and measurements of VAT, EAT, and LF were performed with a 64-multislice $\mathrm{CT}$ and associated workstation (LightSpeed VTC; GE, Milwaukee, WI). VAT was measured on a single abdominal CT section at the level of fourth lumbar vertebra, and EAT was measured from the mid left atrium to the left ventricular apex interpolating $10-\mathrm{mm}$ thick slices with an attenuation of -190 to -30 Hounsfield Units, as described before. ${ }^{16}$ The liver and spleen attenuation (in Hounsfield Unit) were measured in several circular areas of interest, and the presence of LF was defined as a liver-to-spleen ratio $<1.1$.

\section{Statistics}

Variables are expressed as mean and SD or median and interquartile range for continuous and percentage for categorical variables. Univariate analyses ( $U$ Mann-Whitney for continuous variables and Pearson $\chi^{2}$ or Fisher exact text for categorical variables) were conducted to explore factors associated with CVD. Backward stepwise multivariable multilevel Poisson regression analyses were conducted to identify factors independently associated with CVD; we included age, BMI, waist circumference, EAT, VAT, LF, current CD4 count, triglycerides, total cholesterol, apolipoprotein A/apolipoprotein B ratio, smoking, hypertension, diabetes mellitus, and cumulative exposure to ART classes (nucleoside reverse transcriptase inhibitor, nonnucleoside reverse transcriptase inhibitor, and protease inhibitor cumulative exposure). Incidence-rate ratios (IRR), $95 \%$ confidence interval $(\mathrm{CI})$, and $P$ values were reported.

The strength of association between different measures of ectopic fat, namely VAT, EAT, and LF (as a continuous variable), was compared using receiver-operating characteristic curves. The significance of the difference of the area 


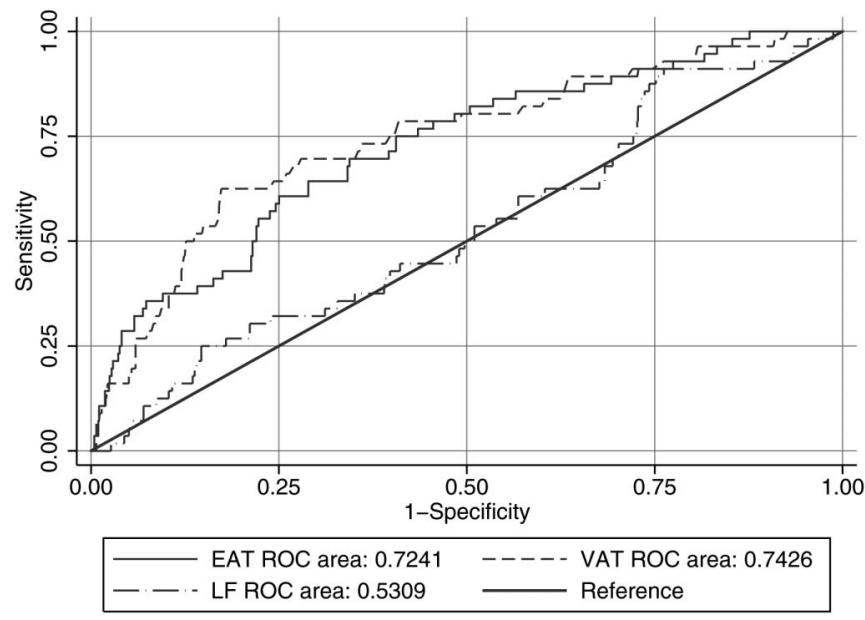

FIGURE 1. Comparison between sensitivity and specificity of EAT and LF measurements.

under the receiver-operating characteristic curves was tested using the roccomp command on Stata. Statistical significance was $P<0.05$. Stata software package, version 10.1 for Mac (Intercooled; StataCorp LP, College Station, TX) was used for the analyses.

\section{RESULTS}

The clinical characteristics of the 583 men included in the study (33 with prior CVD events) are shown in Table 1. Prior CVD events were as follows: MI in 27 patients, primary angioplasty in 3 patients, and stroke in 3 patients.

CVD events occurred with a mean of 12.7 months (range: 1-30.2) before ectopic fat measurements. Patients with previous CVD were older, had a significantly greater waist circumference and BMI, and a higher prevalence of mixed form lipodystrophy but lower prevalence of lipoatrophy than subjects without prior CVD. VAT and EAT were significantly larger in patients with prior CVD compared with those without CVD ( $P<0.0001$ for both), though the prevalence of LF was not different between groups. HOMA-IR and apolipoprotein $\mathrm{A} /$ apolipoprotein $\mathrm{B}$ ratio, also were higher in patients with prior CVD $(P=0.031$ and $P=0.040$, respectively).

Factors independently associated with CVD in multivariable analyses were age (IRR $=1.07,95 \% \mathrm{CI}$ : 1.02 to 1.12 , $P=0.004)$, smoking (IRR $=2.70,95 \% \mathrm{CI}: 1.22$ to $6.01, P=$ $0.015), \mathrm{CDC}$ group $\mathrm{C}(\mathrm{IRR}=3.09,95 \% \mathrm{CI}$ : 1.41 to $6.76, P=$ $0.005)$, EAT $(\operatorname{IRR}=1.13,95 \%$ CI: 1.04 to $1.24, P=0.003$, per $10 \mathrm{~cm}^{3}$ ), LF (IRR $=1.17,95 \% \mathrm{CI}: 1.04$ to $1.32, P=$ 0.008 , per 0.1 increase), and VAT (IRR $=1.05,95 \% \mathrm{CI}$ : 1.00 to $1.10, P=0.028$, per $10 \mathrm{~cm}^{3}$ ).

As shown in Figure 1, EAT and VAT seemed to have a closer association than LF with CVD, although there was no statistical difference between the areas under the curve of these variables $(P=0.325)$. EAT and VAT were associated with previous CVD with the same sensitivity and specificity $(P=0.578)$.

\section{DISCUSSION}

In this cross-sectional sample of men with HIV infection on a stable ART regimen, age, smoking, CDC group C, EAT, and VAT - as measures of ectopic adiposity - were independently associated with prevalent CVD, whereas measures of total (BMI) and regional (waist circumference) adiposity were not. Although this is a retrospective study, it provides a glance into factors that may help improve cardiovascular risk assessment in HIV. Recently, the role of general (BMI) and regional (waist circumference) adiposity measures as a marker of CVD risk in the general population has been criticised. ${ }^{17}$ Measures of ectopic fat, however, have emerged as new important markers of risk. ${ }^{4-10}$ LF seemed to be less sensitive and specific to predict prior CVD than EAT and VAT. This may reflect that CT assessment of LF is less accurate than CT assessment of VAT and EAT assessing relative density of the liver compared with the spleen rather that a direct volume measurement.

EAT is associated with cardio metabolic risk factors, ${ }^{16,18-20}$ and it releases several inflammatory cytokines that might influence the process of atherosclerosis in the coronary arteries. $^{21,22}$ In a population study, EAT was associated with incident cardiovascular events independent of BMI and other risk factors. ${ }^{10}$

The impact of intrabdominal accumulation of adipose tissue in patients with HIV infection as a marker of cardiovascular risk has been studied in connection with the lipodystrophy syndrome. ${ }^{23}$ Interest in EAT has only recently emerged, and this measure of ectopic adiposity has been associated with cardio metabolic risk factors ${ }^{11}$ and subclinical atherosclerosis. ${ }^{12-24}$ Although prospective studies will be necessary to confirm our observations, our findings suggest an association between EAT and CVD in HIV men similar to what has been reported in the general population. ${ }^{10}$ Of interest, the EAT volume we measured in patients with prior CVD was similar to that of a North American cohort of HIV patients reported by Lo et al, ${ }^{11}$ whereas that of subjects without prior CVD was comparable with HIV-negative patients in the same study. As discussed elsewhere, EAT can be measured on the same chest CT images obtained for screening of subclinical atherosclerosis in HIV patients, ${ }^{24}$ without adding time and radiation exposure to the study. EAT can be measured also by transthoracic echocardiography, ${ }^{25}$ although the measurements are less reproducible than with chest $\mathrm{CT}$ or magnetic resonance.

Our study has several limitations, due to its retrospective nature and the measurement of EAT after the occurrence of CVD events; furthermore, this study included only men infected with HIV, and we cannot exclude a selection bias of patient referred to the metabolic clinic. Therefore, caution should be taken to attribute to EAT and/or VAT a causative role in the genesis of CVD in HIV patients before prospective studies are completed. Thus researchers should continue to investigate the role of ectopic fat measurements that may ultimately allow physicians to better discriminate patients at increased risk of events and in need of more intense riskreduction programs.

\section{REFERENCES}

1. Pouliot MC, Despres JP, Lemieux S, et al. Waist circumference and abdominal sagittal diameter: best simple anthropometric indexes of abdominal visceral adipose tissue accumulation and related cardiovascular risk in men and women. Am J Cardiol. 1994;73:460-468. 
2. Johnson D, Prud'homme D, Despres JP, et al. Relation of abdominal obesity to hyperinsulinemia and high blood pressure in men. Int $J$ Obes Relat Metab Disord. 1992;16:881-890.

3. Lemieux I, Pascot A, Prud'homme D, et al. Elevated C-reactive protein: another component of the atherothrombotic profile of abdominal obesity. Arterioscler Thromb Vasc Biol. 2001;21:961-967.

4. Kissebah AH. Intra-abdominal fat: is it a major factor in developing diabetes and coronary artery disease? Diabetes Res Clin Pract. 1996; 30:25-30.

5. Macor C, Ruggeri A, Mazzonetto P, et al. Visceral adipose tissue impairs insulin secretion and insulin sensitivity but not energy expenditure in obesity. Metabolism. 1997;46:123-129.

6. Rondinone CM. Adipocyte-derived hormones, cytokines, and mediators. Endocrine. 2006;29:81-90.

7. Rabkin SW. Epicardial fat: properties, function and relationship to obesity. Obes Rev. 2007;8:253-261.

8. Mazurek T, Zhang L, Zalewski A, et al. Human epicardial adipose tissue is a source of inflammatory mediators. Circulation. 2003;108:2460-2466.

9. Baker AR, Harte AL, Howell N, et al. Epicardial adipose tissue as a source of nuclear factor-kappab and c-jun n-terminal kinase mediated inflammation in patients with coronary artery disease. J Clin Endocrinol Metab. 2009;94:261-267.

10. Ding J, Hsu FC, Harris TB, et al. The association of pericardial fat with incident coronary heart disease: the Multi-Ethnic Study of Atherosclerosis (MESA). Am J Clin Nutr. 2009;90:499-504.

11. Lo J, Abbara S, Rocha-Filho JA, et al. Increased epicardial adipose tissue volume in HIV-infected men and relationships to body composition and metabolic parameters. AIDS. 2010;24:2127-2135.

12. Iacobellis G, Pellicelli AM, Sharma AM, et al. Relation of subepicardial adipose tissue to carotid intima-media thickness in patients with human immunodeficiency virus. Am J Cardiol. 2007;99:1470-1472.

13. Kingsley L, Smit E, Riddler S, et al. Prevalence of lipodystrophy and metabolic abnormalities in the Multicenter Cohort Study (MACS) [abstract 538]. Presented at: Program and abstracts of the Eight Conference on Retroviruses and Opportunistic Infections; February 2001; Chicago, IL.

14. Law MG, Friis-M_ller N, El-Sadr WM, et al. The use of the Framingham equation to predict myocardial infarctions in HIV-infected patients: comparison with observed events in the D:A:D Study. HIV Med. 2006;7:218-230.
15. Tunstall-Pedoe H, Kuulasmaa K, Amouyel P, et al. Myocardial infarction and coronary deaths in the World Health Organization MONICA Project: registration procedures, event rates, and case-fatality rates in 38 populations from 21 countries in four continents. Circulation. 1994; 90:583-612.

16. Gorter PM, van Lindert AS, de Vos AM, et al. Quantification of epicardial and peri-coronary fat using cardiac computed tomography; reproducibility and relation with obesity and metabolic syndrome in patients suspected of coronary artery disease. Atherosclerosis. 2008;197:896-903.

17. The Emerging Risk Factors Collaboration. Separate and combined associations of body-mass index and abdominal adiposity with cardiovascular disease: collaborative analysis of 58 prospective studies. Lancet. 2011; 377:1085-1095.

18. Wang TD, Lee WJ, Shih FY, et al. Relations of epicardial adipose tissue measured by multidetector computed tomography to components of the metabolic syndrome are region-specific and independent of anthropometric indexes and intraabdominal visceral fat. J Clin Endocrinol Metab. 2009;94:662-669.

19. de Vos AM, Prokop M, Roos CJ, et al. Peri-coronary epicardial adipose tissue is related to cardiovascular risk factors and coronary artery calcification in postmenopausal women. Eur Heart J. 2008;29:777-783.

20. Wang CP, Hsu HL, Hung WC, et al. Increased epicardial adipose tissue (EAT) volume in type 2 diabetes mellitus and association with metabolic syndrome and severity of coronary atherosclerosis. Clin Endocrinol (Oxf). 2009:70:876-882.

21. Sacks HS, Fain JN. Human epicardial adipose tissue: a review. Am Heart J. 2007;153:907-917.

22. Shibasaki I, Nishikimi T, Mochizuki Y, et al. Greater expression of inflammatory cytokines, adrenomedullin, and natriuretic peptide receptor-C in epicardial adipose tissue in coronary artery disease Regul Pept. 2010;165:210-217.

23. Barbaro G. Metabolic and cardiovascular complications of highly active antiretroviral therapy for HIV infection. Curr HIV Res. 2006;4:79-85.

24. Guaraldi G, Scaglioni R, Zona S, et al. Epicardial adipose tissue is an independent marker of cardiovascular risk in HIV-infected patients. AIDS. 2011;25:1199-1205.

25. Eroglu S, Sade LE, Yildirir A, et al. Epicardial adipose tissue thickness by echocardiography is a marker for the presence and severity of coronary artery disease. Nutr Metab Cardiovasc Dis. 2009;19:211-217. 